\section{Fiberoptic surgery by ultrabright lamp light}

\author{
Jeffrey M. Gordon, ${ }^{\mathrm{a}, \mathrm{b}, *}$ Ruthy Shaco-Levy, ${ }^{\mathrm{c}}$ \\ Daniel Feuermann, ${ }^{a}$ Jared Ament, ${ }^{d}$ and Solly Mizrahid \\ ${ }^{\mathrm{a} B e n-G u r i o n}$ University of the Negev, Jacob Blaustein \\ Institutes for Desert Research, Department of Solar Energy \\ and Environmental Physics, Sede Boqer Campus \\ 84990, Israel \\ ${ }^{\mathrm{b}}$ Ben-Gurion University of the Negev, The Pearlstone \\ Center for Aeronautical Engineering Studies, Department of \\ Mechanical Engineering, Beersheva 84105, Israel \\ 'Ben-Gurion University of the Negev, Department of \\ Pathology, Soroka Medical Center and Faculty of Health \\ Sciences, Beersheva 84105, Israel \\ ${ }^{\mathrm{d} B e n-G u r i o n}$ University of the Negev, Department of \\ Surgery A, Soroka Medical Center and Faculty of Health \\ Sciences, Beersheva 84105, Israel
}

Abstract. We report the first realization of interstitial surgery by ultrabright lamp light, on the kidneys and livers of live animals. A high-flux optic concentrates lamp emissions into an optical fiber for power delivery inside the body. The trials reveal surgical efficacy comparable to corresponding laser fiber optic treatments, as well as pronounced delayed tissue death. $\odot 2006$ Society of Photo-Optical Instrumentation Engineers. [DOI: 10.1117/1.2363354]

Keywords: ultrabright lamp light; interstitial surgery; optical fiber; efficacy.

Paper 06175LR received Jun. 29, 2006; revised manuscript received Aug. 15, 2006; accepted for publication Aug. 25, 2006; published online Oct. 24, 2006.

Laser fiberoptic surgery is a minimally invasive technique for the rapid and localized necrosis of growths in internal organs. ${ }^{1-4}$ The considerable cost of laser surgical systems prompts the quest for less expensive noncoherent photonic alternatives, especially since the key essential laser characteristic often is immense power density rather than monochromaticity or coherence.

Recently, we demonstrated that highly concentrated solar radiation can generate the same degree, rate, and type of photothermal tissue damage as lasers. ${ }^{5}$ However, its value is restricted by the ephemeral nature of sunlight. Here, we demonstrate the first exploitation of artificial noncoherent light for photothermal surgery on live rats, with an inexpensive device as efficacious as many surgical lasers.

A compact tabletop optical system was developed ${ }^{6}$ [Fig. 1(a)] that reconstitutes the power density from the radiant plasma of a commercial ultrabright lamp inside an optical fiber that delivers light inside the body [Figs. 1(b) and 1(c)]. The light source was a $150-\mathrm{W}$ xenon short-arc discharge lamp, ${ }^{7}$ with a $2.0-\mathrm{mm}$ electrode gap, which emits an approximately $6000-\mathrm{K}$ graybody spectrum enhanced by strong xenon emission peaks at wavelengths from 800 to $1000 \mathrm{~nm}$. This source is suitable for photothermal surgery because 1 . it subsumes visible and near-infrared wavelengths where tissue optical penetration is greatest, ${ }^{1,2}$ and 2 . its brightness is high enough to provide the required power density, a feat unattain-

*Tel: 9728659 6923; E-mail: jeff@bgu.ac.il able with other light sources such as light-emitting diodes or incandescent lamps.

A dual-mirror compact aplanatic optic [Fig. 1(a)] concentrates lamp emissions into an optical fiber. The (silvered specular) reflector contours enable high-flux transfer by eliminating spherical aberration and coma for a relatively small light source [Fig. 1(a)], and are essentially achromatic. The analytic equations that describe the reflectors were reported in Ref. 8. A hemispherical mirror recycles lamp emissions and heightens delivered power by about 50\%.

The fiber had a quartz core diameter of $1.0 \mathrm{~mm}$, a teflon cladding, and a numerical aperture $(N A)$ of 0.66 . The fact that high power density mandates high $N A$, i.e., large delivery angle, follows from the principle of phase space conservation, ${ }^{9}$ and limits effective lamp surgery to the fiber being inside the organ or maintained close to its surface. The largest $N A$ value in commercially available fibers that are also transmissive over the lamp's emission spectrum (350 to $2500 \mathrm{~nm}$ ) is 0.66 . A pragmatic benefit of large-angle fiber emission is that lamp surgery does not engender the risk of eye injury to the surgical team.

The fiber was fit snugly into a protective Pyrex sleeve, via which light was injected to the organs. A sleeve thickness of about $1 \mathrm{~mm}$ separated the fiber tip from the irradiated tissue, such that the spot size on the tissue was around $2 \mathrm{~mm}$.

Recent ex-vivo lamp surgery experiments ${ }^{6}$ guided our choice of irradiation times and flux values for generating immediate necrosis of the order of hundreds of $\mathrm{mm}^{3}$ : a compromise between the large lesions often needed in human surgery and the small size of rat kidney and liver. Lamp surgery with irradiation times varying from 60 to $240 \mathrm{~s}$ and radiative input from 1.7 to $2.1 \mathrm{~W}$ was conducted on the livers and kidneys of 13 anesthetized healthy adult rats divided into groups for histopathology examination at 0 (immediate group), 24, and 48 (delayed group) hours after surgery. Input power was purposely limited toward minimizing the risks of carbonization while producing sizable necrosis with relatively short exposures. Optical losses from the light source to the net delivered radiative power were assessed by ray-trace simulation in Ref. 8. Given the additional inefficiency in converting electricity to utilizable visible and near-IR light of sufficient brightness, ${ }^{7}$ lamp nominal power ratings of around $100 \mathrm{~W}$ and higher are needed for effective photothermal surgery.

Healthy adult rats of body mass 300 to $400 \mathrm{~g}$ were used in all experiments. The targeted organs were exposed to view with a longitudinal midline scalpel abdominal incision. Experiments were approved by the Institutional Animal Care and Use Committee of the Soroka Medical Center, Ben-Gurion University, Beersheva, Israel, and the surgeons underwent the health protection program for handling research animals. All organs were irradiated in regions that would not interfere with large veins, thus avoiding major vascular thrombosis that could otherwise contribute to lesion size.

Both the thick and thin lobes of the liver were irradiated in all procedures. Only one kidney was treated in each of the rats in the delayed group to avoid complete renal failure. For rats in the immediate group, a single exposure was applied to both kidneys. Those animals intended for delayed pathology were revived, and functioned without complication.

1083-3668/2006/11(5)/050509/2/\$22.00 @ 2006 SPIE 

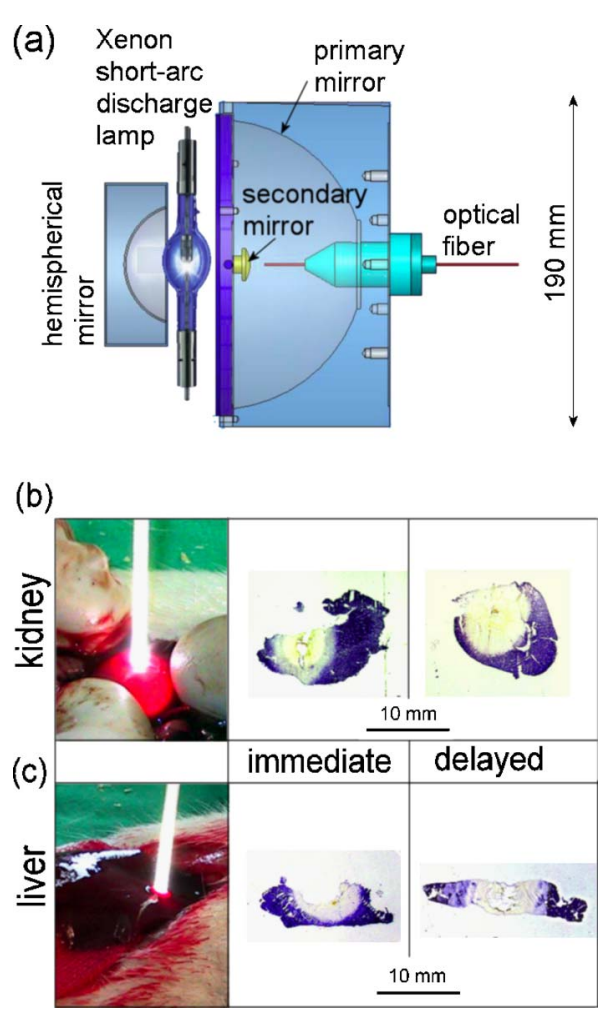

Fig. 1 Optics, surgical procedure, and pathology on living rats. (a) Drawing of the compact high-flux optic that concentrates ultrabright lamp emissions into an optical fiber. Intense noncoherent (white) light being delivered to the center of (b) the kidney and (c) liver of a live anesthetized rat, with NADH-diaphorase stain pathology for sections from rats in the immediate and 24 -h groups. Irradiation of $1.9 \mathrm{~W}$ was applied for 60 and $90 \mathrm{~s}$ to these kidneys and livers, respectively. Viable tissue stains blue (dark). Nonviable (necrosed) tissue appears transparent (color online only).

Liver and kidney pathology for all procedures was investigated with NADH-diaphorase stain. Pathology for the 24 and $48 \mathrm{~h}$ groups was also performed with haematoxylin-andeosin ( $\mathrm{H}$ and $\mathrm{E})$ stain for independent confirmation. The $\mathrm{H}$ and $\mathrm{E}$ technique was constrained to the delayed group, because it was previously shown to lead to dramatic underestimates for immediate pathology, the shortfall being mitigated when histological examination is postponed for $24 \mathrm{~h}$ or longer. ${ }^{10}$

Pathology revealed the coagulative and ablative tissue transformations characteristic of laser surgery ${ }^{1-4}$ [Figs. 1(b) and 1(c)]. Lesion volumes were calculated from the lateral and longitudinal cross sectional diameters observed in the pathology slides, and varied from 100 to $800 \mathrm{~mm}^{3}$ with shapes that were roughly hemiellipsoidal for liver and fully ellipsoidal for kidney. In samples where the necrosis traversed the entire organ, lesion volume was estimated from the linear dimensions of the actual asymmetric coagulated region. The extent of necrosis was distinctly larger after $24 \mathrm{~h}$ [Figs. 1(b) and 1(c)] but did not increase further at $48 \mathrm{~h}$.

For a given time after surgery, the volume of dead tissue was approximately proportional to both exposure time and input power. Surgical efficacy $E$ (lesion volume per delivered light energy) is then a suitable figure of merit for assessing and comparing results. Since no significant differences were discerned between animals in the 24 and $48 \mathrm{~h}$ groups, those results were lumped into a single delayed category.

We found $E_{\text {kidney }}=1.4 \pm 0.4$ and $E_{\text {liver }}=1.0 \pm 0.2 \mathrm{~mm}^{3} / \mathrm{J}$ immediately after irradiation - values commensurate with corresponding results for laser fiberoptic ${ }^{1-4}$ and solar ${ }^{5}$ surgery. For the delayed groups, $E_{\text {kidney }}=2.3 \pm 0.4$ and $E_{\text {liver }}$ $=2.7 \pm 1.2 \mathrm{~mm}^{3} / \mathrm{J}$. Some lesions traversed the entire liver lobe [Fig. 1(c)]. They represent a lower bound for surgical efficacy, and account for the relatively large variance in $E_{\text {liver. }}$.

When induced photothermally, delayed tissue death is an athermal process of sizable degree ${ }^{11-14}$ The phenomenon is only sparsely documented for livers, ${ }^{10-13}$ and the understanding of the underlying mechanisms is incomplete. ${ }^{12}$ There are no analogous results of which we are aware for kidneys.

The comparable efficacy of lamp and laser procedures stems from their sharing similar optical and biophysical properties. ${ }^{1-3,5,6}$ As such, the virtues of lamp surgery relative to hot-wire techniques, radiofrequency ablation, and cryoablation should be the same as those for laser surgery compared to the same methods. ${ }^{14}$

The confluence of maximum-flux optics and the suitability of ultrabright lamp light for interstitial photothermal surgery bodes well for the feasibility of a photonic tool that is potentially far less expensive than corresponding laser surgical systems and offers comparable effectiveness.

\section{References}

1. A. Katzir, Lasers and Optical Fibers in Medicine, Academic Press, San Diego (1993).

2. A. J. Welch and M. J. C. van Gemert, Optical-Thermal Response of Laser-Irradiated Tissue, Plenum Press, New York (1995).

3. R. M. Verdaasdonk and F. P. van Swol, "Laser light delivery systems for medical applications," Phys. Med. Biol. 42, 869-894 (1997).

4. J. Heisterkamp, R. van Hillegersberg, E. Sinofsky, and J. N. M. Ijzermans, "Heat-resistant cylindrical diffuser for interstitial laser coagulation: comparison with the bare-tip fiber in a porcine liver model," Lasers Surg. Med. 20, 304-309 (1998).

5. J. M. Gordon, D. Feuermann, M. Huleihil, S. Mizrahi, and R. ShacoLevy, "Surgery by sunlight on live animals," Nature (London) 424, 510 (2003).

6. D. Feuermann, J. M. Gordon, and T. W. Ng, "Photonic surgery with noncoherent light," Appl. Phys. Lett. 88, 114104 (2006).

7. Hamamatsu Corp., Technical Brochures, Shimokanzo, Toyooka Village, Iwata-gun, Shizuoka-ken 438-0193, Japan (2004).

8. D. Nakar, D. Feuermann, and J. M. Gordon, "Aplanatic near-field optics for efficient light transfer," Opt. Eng. 45, 030502 (2006).

9. R. Winston, J. C. Miñano, and P. Benítez, Nonimaging Optics, Elsevier Academic Press, Oxford (2005).

10. R. Shaco-Levy, J. M. Gordon, D. Feuermannm, M. Huleihil, and S. Mizrahi, "On appropriate pathology for photothermal surgery," Lasers Surg. Med. 35, 28-34 (2004).

11. Y. Fujitomi, K. Kashima, S. Ueda, Y. Yamada, H. Mori, and Y. Uchida, "Histopathological features of liver damage induced by laser ablation in rabbits," Lasers Surg. Med. 24, 14-23 (1999).

12. M. Nikfarjam, V. Muralidharan, and C. Christophi, "Mechanisms of focal heat destruction of liver tumors," J. Surg. Res. 127, 208-223 (2005).

13. J. M. Gordon, R. Shaco-Levy, D. Feuermann, M. Huleihil, and S. Mizrahi, "Photothermally induced delayed tissue death," J. Biomed. Opt. 11, 030504 (2006).

14. J. T. De Sanctis, S. N. Goldberg, and P. R. Mueller, "Percutaneous treatment of hepatic neoplasms: a review of current techniques," Cardiovasc. Intervent Radiol. 21, 273-296 (1998). 